\title{
BANYAN. VI. Discovery of a companion at the brown dwarf/planet-mass limit to a Tucana-Horologium M dwarf
}

\author{
Étienne Artigau ${ }^{1}$, Jonathan Gagné ${ }^{1}$, Jacqueline Faherty ${ }^{2}$, Lison Malo ${ }^{3}$, Marie-Eve Naud $^{1}$, \\ René Doyon ${ }^{1}$, David Lafrenière ${ }^{1}$, Yuri Beletsky ${ }^{4}$ \\ Send correspondence to artigau@astro.umontreal.ca
}

\begin{abstract}
We report the discovery of a substellar companion to 2MASS J02192210-3925225, a young M6 $\gamma$ candidate member of the Tucana-Horologium association $(30-40 \mathrm{Myr})$. This L4 $\gamma$ companion has been discovered with seeing-limited direct imaging observations; at a $4^{\prime \prime}$ separation $(160 \mathrm{AU})$ and a modest contrast ratio, it joins the very short list of young low-mass companions amenable to study without the aid of adaptive optics, enabling its characterization with a much wider suite of instruments than is possible for companions uncovered by high-contrast imaging surveys. With a model-dependent mass of $12-15 \mathrm{M}_{\mathrm{Jup}}$, it straddles the boundary between the planet and brown dwarf mass regimes. We present near-infrared spectroscopy of this companion and compare it to various similar objects uncovered in the last few years. The J0219-3925 system falls in a sparsely populated part of the host mass versus mass ratio diagram for binaries; the dearth of known similar companions may be due to observational biases in previous low-mass companion searches.
\end{abstract}

Subject headings:

\section{Introduction}

The spectacular discoveries brought on by highcontrast imaging of exoplanets around nearby stars (e.g., HR8799's system, Marois et al. 2008, 2010; $\beta$ Pictoris b, Lagrange et al. 2010; Fomalhaut's companion, Kalas et al. 2008 ) eclipse a fact that is often overlooked: most of the region around a star where a planet would be gravitationally bound for Gyrs is readily accessible to seeing-limited observations for nearby stars. A handful of planetary-mass objects have indeed been uncovered in wide-field surveys, such as GU

\footnotetext{
${ }^{1}$ Institut de Recherche sur les Exoplanètes (IREx), Département de Physique, Université de Montréal, C.P. 6128, Succ. Centre-Ville, Montréal, QC, H3C 3J7, Canada

${ }^{2}$ Department of Terrestrial Magnetism, Carnegie Institution of Washington, Washington, DC 20015, USA

${ }^{3}$ Canada-France-Hawaii Telescope Corporation, 65-1238 Mamalahoa Highway, Kamuela, HI 96743, USA

${ }^{4}$ Las Campanas Observatory, Carnegie Institution of Washington, Colina el Pino, Casilla 601, La Serena, Chile
}

Psc b and Ross 458(AB)c, respectively 2000 and $1200 \mathrm{AU}$ from their hosts (Goldman et al. 2010; Burningham et al. 2011; Naud et al. 2014), and the presence of companions on such wide orbits presents a significant challenge to planetary formation models.

These companions are on orbits too large for in situ formation by either core accretion (Pollack et al. 1996; Alibert et al. 2005) or gravitational instability within protoplanetary disks (Cameron 1978; Boss 1997). Vorobyov (2013) established that wide companions may form in wide orbits within gravitationally unstable protoplanetary disks, but only around massive $\left(>0.7 \mathrm{M}_{\odot}\right)$ hosts with massive $\left(>0.2 \mathrm{M}_{\odot}\right)$ protoplanetary disks, and cannot account for the discovery of such companions around $\mathrm{K}$ stars or later-type hosts. Turbulent fragmentation of a pre-stellar core (Padoan \& Nordlund 2002) is a viable alternative to explain the existence of such systems. Outward migration through planet-planet interaction is also plausible, but remains to be tested 
as a credible mechanism at such separations and mass ratios (Veras et al. 2009). Statistical analyses of the mass, age and separation of planets uncovered through high-contrast imaging campaigns suggest that these companions constitute a low-mass tail of the brown dwarf distribution and are therefore the results of disk or cloud fragmentation. The dearth of low-mass $\left(<5 \mathrm{M}_{J}\right)$ companions found by direct imaging surveys with adaptive optics ( $\mathrm{AO}$ ) can be explained in that context (Brandt et al. 2014). This analysis mostly contrains the occurence of companions out to 100$200 \mathrm{AU}$; whether this paucity of companions holds for more distant brown dwarfs and planetary-mass companions remains to be determined in a statistical framework. Overall, much work needs to be done to understand the origin and demographics of these distant companions, both theoretically and observationally.

These distant companions have distinct advantages compared to either isolated planetarymass objects or planets detected through AO surveys. The presence of a host star allows a crosscalibration of the properties of the planet (parallax, mass, age, metallicity, membership to a young group), and the projected separation to that host is sufficient to allow direct study through means not compatible with extreme-AO, such as accurate spectro-photometry, high-resolution spectroscopy, optical imaging, etc. Indeed, the detailed analysis performed with intermediate-resolution spectrophotometry on GU Psc b and Ross 458(AB)c will be impossible to obtain in the near future for exoplanets uncovered by AO surveys.

As distant companions of nearby young stars provide important benchmarks to understand selfluminous gas giants, we undertook various seeinglimited observations using the SIMON nearinfrared spectro-imager (Doyon et al. 2000) at the CTIO-1.5 m telescope and GMOS-S at Gemini South in order to identify such new objects through their distinctive far-red and near-infrared colors. We report here a first discovery from this survey; a co-moving companion to 2MASS J02192210-3925225 (J0219-3925), an M6 $\gamma$ candidate member of the Tucana-Horologium association (THA) that has a low-gravity L4 $\gamma$ companion.

The survey that led to this discovery is described in section 2. The discovery and photo- metric follow-ups of the companion and its host are described in section 3. Results are described in section 4 and discussed in section 5

\section{The survey}

J0219-3925 has been observed as part of a survey of 300 stars conducted at the CTIO- $1.5 \mathrm{~m}$ telescope with the SIMON. This survey was undertaken following the discovery of the planetarymass companion around the M3 AB Doradus member GU Psc (Naud et al. 2014), in an attempt to identify additional comparable planetary-mass companions and assess their overall frequency. The target sample consists of both confirmed and strong members of young moving groups within 70 pc (< 120 Myr; $\beta$ Pictoris, AB Doradus, THA, Columba, Carina and Argus), and it was assembled from objects in Malo et al. (2013) and Gagné et al. (2015). For each star, the Bayesian Analysis for Nearby Young AssociatioNs II tool (BANYAN II; Gagné et al. 2014c) provided a statistical distance estimate, allowing us to derive a projected distance for putative companions.

By combining SIMON J-band imaging with WISE photometry (Wright et al. 2010), we identified objects that had a similar position to brown dwarfs or known very-low gravity $L$ dwarfs in an $\mathrm{M}_{W 1}$ vs $J-W 2$ diagram, but that did not have optical digitized sky survey (DSS) counterparts.

In a way similar to the survey that allowed the discovery of GU Pscb, candidates were then followed-up with Gemini-South using deep $i$ and $z$ band imaging to confirm the very red $i-z$ color expected for an L or T dwarf. The selection methods will be detailed in a future paper (Artigau et al. in preparation), as refinement of selection methods and follow-up of candidates are still ongoing.

As the SIMON observations have significantly better resolution than 2 MASS $\left(\sim 1^{\prime \prime}\right.$ versus $\sim 2^{\prime \prime}$ full-width at half maximum) and are significantly deeper $(10 \sigma$ at $J \sim 18)$, they provide an opportunity to identify relatively tight $\left(2-6^{\prime \prime}\right)$ overlooked companions. We performed a radial profile subtraction on all primaries and visually inspected the residuals. Through this process, a single candidate was identified, at an angular distance of about $4^{\prime \prime}$ from the M6 star J0219-3925. No other star within our sample presented a similar candidate companions within in a $2-6^{\prime \prime}$ annulus down 
to a contrast of $\Delta J<5$.

\section{Observation and reduction}

\subsection{Imaging}

The J-band discovery dataset of J0219-3925 was obtained with SIMON on 2013 November 5 at the CTIO, and follow-up $H$ and $K_{s}$-band imaging were obtained on 2014 February 2, with the same instrument and telescope. For all 3 imaging sequences, we employed a 4-point dither pattern along the corners of a $2^{\prime} \times 2^{\prime}$ square. At each dither position, 15 images $(J)$ and 5 images $(H$ and $K_{s}$ ) were taken. All sequences used a $30 \mathrm{~s}$ per-frame exposure time. The images were skysubtracted using a sky frame constructed from the median combination of all science images taken on that night within that band. Flat-fielding was performed with a flat constructed from images of a flat screen. The astrometric solution was performed using a cross-match of the 2MASS catalog with field stars. All images were registered and median-combined to produce the final science frame.

The contrast ratio between the two components was determined by performing a PSF fitting of the two J0219-3925 components using two isolated bright and nearby field stars $(<2.5, J>13.5)$ as input PSFs. The magnitude of J0219-3925 B was then determined from the 2MASS magnitude of its host and its contrast ratio within each nearinfrared bandpass. The presence of the companion is unlikely to have significantly affected the 2MASS magnitudes of its host, as it contributes only 2 to $4 \%$ of the total integrated flux and is resolved $(\sim 2$ FWHM).

Upon discovery of a possible faint companion to J0219-3925, we noticed that images of that field usable for science were present in the Gemini science archive (See Figure 1). GMOS-South ( $i$ and $g$-band) and Flamingos-II ( $J$-band) spectroscopic acquisition images of the host star have been obtained as follow-up observations of the BANYAN All-Sky Survey (BASS; Gagné et al. 2015). The $\Delta i=6.22$ contrast and non-detection in $g(\Delta g>$ 5.5) pointed toward an object much redder than the mid-M host, prompting for a dedicated spectroscopic follow-up of the companion. Inspection of the $K$-band 2MASS images taken in 1999 shows that the companion is marginally detected
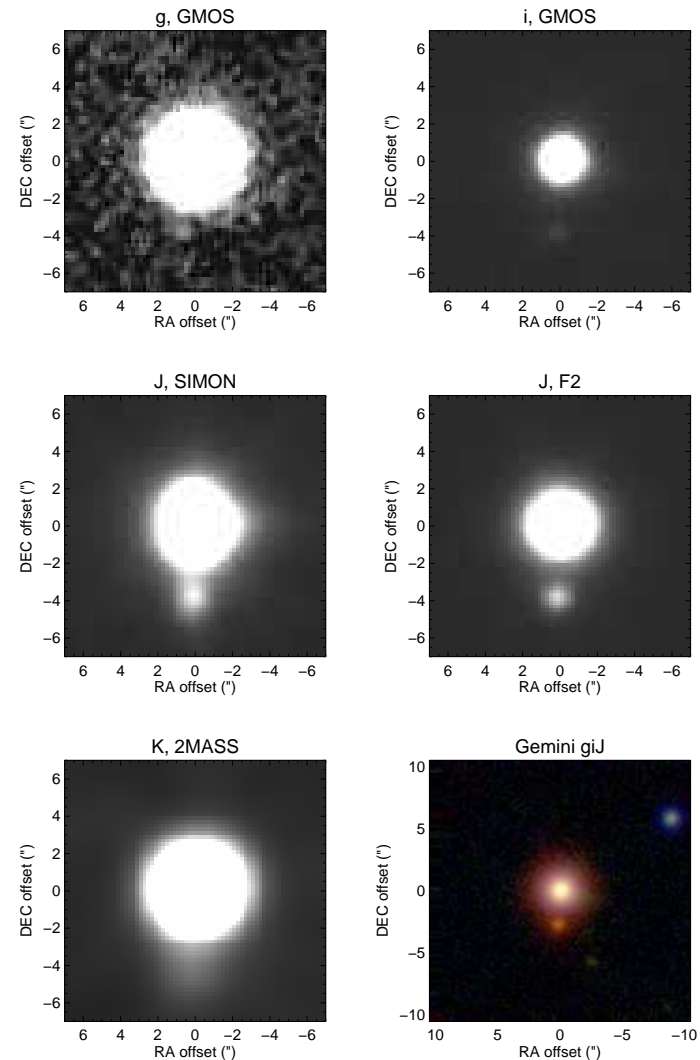

Fig. 1.- J0219-3925 system in SIMON, Flamingos 2 (F2), GMOS and 2MASS imagery. Despite modest constraints provided by the 2MASS image on the position of J0219-3925 B, the $\sim 15$ yr delay between these archival observations and our discovery images provides significant constraints on the common proper motion (see section 4.4). For all images, East is left, North is up. The color image is a combination of $g, i$ and F2 $J$-band images respectively coded as blue, green and red with arbitrary scaling. One readily sees that J0219$3925 \mathrm{~B}$ is much redder than its host. 
Table 1: Imaging and spectroscopy datasets for the J0219-3925 system

\begin{tabular}{|c|c|c|c|c|c|}
\hline Instrument & Date & Filter & $\begin{array}{c}\text { Number } \\
\text { of exposures }\end{array}$ & $\begin{array}{c}\text { Per-frame } \\
\text { exposure time }\end{array}$ & $\begin{array}{c}\text { Comment } \\
\text { where applicable } \\
\text { Gemini program ID }\end{array}$ \\
\hline GMOS-S & $17 / 09 / 2012$ & $g$ & 1 & $10.5 \mathrm{~s}$ & $\begin{array}{c}\text { Spectroscopy acquisition } \\
\text { GS-2012B-Q-70 }\end{array}$ \\
\hline GMOS-S & $02 / 11 / 2012$ & $i$ & 1 & $10.5 \mathrm{~s}$ & $\begin{array}{c}\text { Spectroscopy acquisition } \\
\text { GS-2012B-Q-70 }\end{array}$ \\
\hline SIMON & $05 / 11 / 2013$ & $J$ & 60 & $30 \mathrm{~s}$ & Discovery imaging \\
\hline SIMON & $12 / 02 / 2014$ & $H$ & 20 & $30 \mathrm{~s}$ & Photometric follow-up \\
\hline SIMON & $12 / 02 / 2014$ & $K_{s}$ & 20 & $30 \mathrm{~s}$ & Photometric follow-up \\
\hline $\mathrm{F} 2$ & $28 / 10 / 2013$ & $J$ & 1 & $5 \mathrm{~s}$ & $\begin{array}{c}\text { Spectroscopy acquisition } \\
\text { GS-2013B-Q-79 }\end{array}$ \\
\hline FIRE & $13 / 02 / 2014$ & $\cdots$ & 2 & $306 \mathrm{~s}$ & J0219-3925, spectroscopy \\
\hline FIRE & $13 / 02 / 2014$ & $\cdots$ & 4 & $909 \mathrm{~s}$ & J0219-3925 B, spectroscopy \\
\hline F2 & $04 / 09 / 2014$ & $J$ & 27 & $54 \mathrm{~s}$ & $\begin{array}{c}\text { Imaging for astrometry } \\
\text { GS-2014B-Q-72 }\end{array}$ \\
\hline
\end{tabular}

and provided valuable constraints on the common proper motion of the pair (see Section 4.4 and Table 3 .

\subsection{Spectroscopy}

On 2014 February 13, we obtained near-infrared spectroscopic observations with the Folded-Port Infrared Echelette (FIRE; Simcoe et al. 2013) at the Magellan 6.5-m telescope. FIRE was used in its high-resolution echellette mode, providing a spectral resolution $R \sim 5000$ continuously over the entire near-infrared domain $(0.8-2.4 \mu \mathrm{m})$. We used a slit width of 0 '! 6 under a 1 .' 2 seeing and an airmass of $1.3-1.6$ (J0219-3925 B) and $1.8-1.9$ (J0219-3925A). The reference star for both objects was observed at the same airmasses. For J0219-3925B, we used an ABBA dither pattern for improved sky subtraction on this relatively faint target, while for the brighter J0219-3925 A and reference star, the two exposures were taken with an $\mathrm{AB}$ dither pattern.

Data reduction was performed using the standard FIREHOSE pipeline. Flat-field correction was performed using flat frames derived from dome flat images and telluric absorption at the time observation was derived from an A0V star (HD 17683) observation taken immediately before (J0219-3925B) and after (J0219-3925A) the science integration. The spectrum of the host star and its companion are compared to field and lowgravity objects of comparable spectral types in Figure 2. The spectrum of J0219-3925 B has an average $\mathrm{S} / \mathrm{N}$ of $\sim 30$ when sampled at an $R=800$ resolution.

\section{Results}

\subsection{Host star properties and membership}

J0219-3925 has been identified as a member of the THA by two teams independently. First, it is part of the 129 new late-type (K3 and later) THA members identified by Kraus et al. 2014; their membership being established by Li and RV measurements, but lack parallaxes and is based on spectroscopic distances. Spectroscopic fitting of optical spectrum and overall SED of J02193925 respectively lead to a spectral type estimate of M4.9 \pm 1.0 and M5.9 \pm 0.3 ; these values are consistent with the near-infrared spectral type of M6 $\gamma$ determined here (see section 4.3). The radial velocity they measure $\left(10.6 \pm 0.7 \mathrm{~km} \mathrm{~s}^{-1}\right)$ agrees within $0.71 \mathrm{~km} \mathrm{~s}^{-1}$ with the expected radial velocity of a THA member in that line of sight. This is below the internal dispersion of THA velocities $\left(\sim 1 \mathrm{kms}^{-1}\right)$ and well below the cutoff in velocity difference between members and non-members in their analysis $\left( \pm 3 \mathrm{~km} \mathrm{~s}^{-1}\right)$.

The host star J0219-3925 was identified in- 
Table 2: Properties of the J02193925 system

\begin{tabular}{|c|c|c|}
\hline & \\
\hline Short name & J0219-3925 & J0219-3925B \\
\hline 2MASS name & \multicolumn{2}{|c|}{ 02192210-3925225 } \\
\hline$\mu_{\alpha}{ }^{a}$ (mas/yr) & \multicolumn{2}{|c|}{$107.37 \pm 2.27$} \\
\hline$\mu_{\delta}^{a}(\operatorname{mas} / \mathrm{yr})$ & \multicolumn{2}{|l|}{$-34.95 \pm 1.65$} \\
\hline Statistical distance ${ }^{b}$ & \multicolumn{2}{|c|}{$39.4 \pm 2.6 \mathrm{pc}$} \\
\hline Position angle & \multicolumn{2}{|c|}{$173.9 \pm 0.2^{\circ}$} \\
\hline Separation & \multicolumn{2}{|c|}{$3.96 \pm 0.02^{\prime \prime}$} \\
\hline Sky-plane separation & \multicolumn{2}{|c|}{$156 \pm 10 \mathrm{AU}$} \\
\hline$B$ & \multicolumn{2}{|c|}{18.50} \\
\hline$\Delta g^{c}$ & \multicolumn{2}{|c|}{$>5.5$} \\
\hline$\Delta i^{c}$ & \multicolumn{2}{|c|}{6.6} \\
\hline$R$ & 15.23 & $\cdots$ \\
\hline$I^{d}$ & $13.42 \pm 0.03$ & .. \\
\hline$J_{2 M a s s}{ }^{e}$ & $11.381 \pm 0.026$ & $\cdots$ \\
\hline$J_{M K O} f$ & $11.321 \pm 0.026$ & $15.54 \pm 0.10$ \\
\hline$H_{2 M a s s} e$ & $10.811 \pm 0.027$ & $\ldots$ \\
\hline$H_{M K O}{ }^{f}$ & $10.855 \pm 0.027$ & $14.63 \pm 0.10$ \\
\hline$K_{2 M a s s}{ }^{e}$ & $10.404 \pm 0.025$ & $\cdots$ \\
\hline$K_{M K O}{ }^{f}$ & $10.444 \pm 0.025$ & $13.82 \pm 0.10$ \\
\hline$W 1^{g}$ & \multicolumn{2}{|c|}{$10.148 \pm 0.023$} \\
\hline$W 2^{g}$ & \multicolumn{2}{|c|}{$9.901 \pm 0.020$} \\
\hline$W 3^{g}$ & \multicolumn{2}{|c|}{$9.614 \pm 0.037$} \\
\hline$v_{\text {rad }}$ & $10.6 \pm 0.7^{\mathrm{g}}$ & $\ldots$ \\
\hline$v \sin i$ & $6.5 \pm 0.4^{\mathrm{g}}$ & $\cdots$ \\
\hline SpT & M6 $\gamma^{\mathrm{h}}$ & $\mathrm{L} 4 \gamma$ \\
\hline EW H $\alpha(\AA)$ & -7.02 & $\cdots$ \\
\hline EW $\operatorname{Li}_{6708}(\AA)$ & 639.9 & $\ldots$ \\
\hline $\mathrm{FeH}_{z}$ & $1.068 \pm 0.001$ & $1.040 \pm 0.002$ \\
\hline $\mathrm{FeH}_{J}$ & $1.07 \pm 0.01$ & $1.20 \pm 0.02$ \\
\hline KI $1.169 \mu \mathrm{m}$ & $0.17 \pm 0.08$ & $0.6 \pm 0.4$ \\
\hline KI $1.177 \mu \mathrm{m}$ & $1.25 \pm 0.06$ & $0.71 \pm 0.32$ \\
\hline KI $1.244 \mu \mathrm{m}$ & $\ldots{ }^{i}$ & $3.0 \pm 0.5$ \\
\hline NaI $1.138 \mu \mathrm{m}$ & $2.58 \pm 0.08$ & $2.4 \pm 0.5$ \\
\hline$H$-Cont & $0.995 \pm 0.001$ & $0.963 \pm 0.001$ \\
\hline $\mathrm{VO}_{z}$ & $1.001 \pm 0.001$ & $1.372 \pm 0.004$ \\
\hline
\end{tabular}

Girard et al. 2011

${ }^{b}$ See Gagné et al. 2014d for a discussion on the definition and the accuracy of the statistical distance.

${ }^{c}$ Only contrasts were derived in $g$ and $i$, and not magnitudes measurements as the images were not taken under photometric conditions.

$d$ DENIS Consortium 2005

Skrutskie et al. 2006

${ }^{f}$ The 2MASS to MKO transform has been determined for J0219-3925 from its FIRE spectrum. The companion's contrast ratio has been determined with SIMON that uses MKO filters. 9 Cutri et al. 2013

hraus et al. 2014 give a M5.9 spectral type from SED fitting and a spectroscopic spectral type of M4.9.

${ }^{i}$ The spectrum of J0219-3925 B around the KI $1.244 \mu \mathrm{m}$ is strongly affected by bad pixels on the science array. 

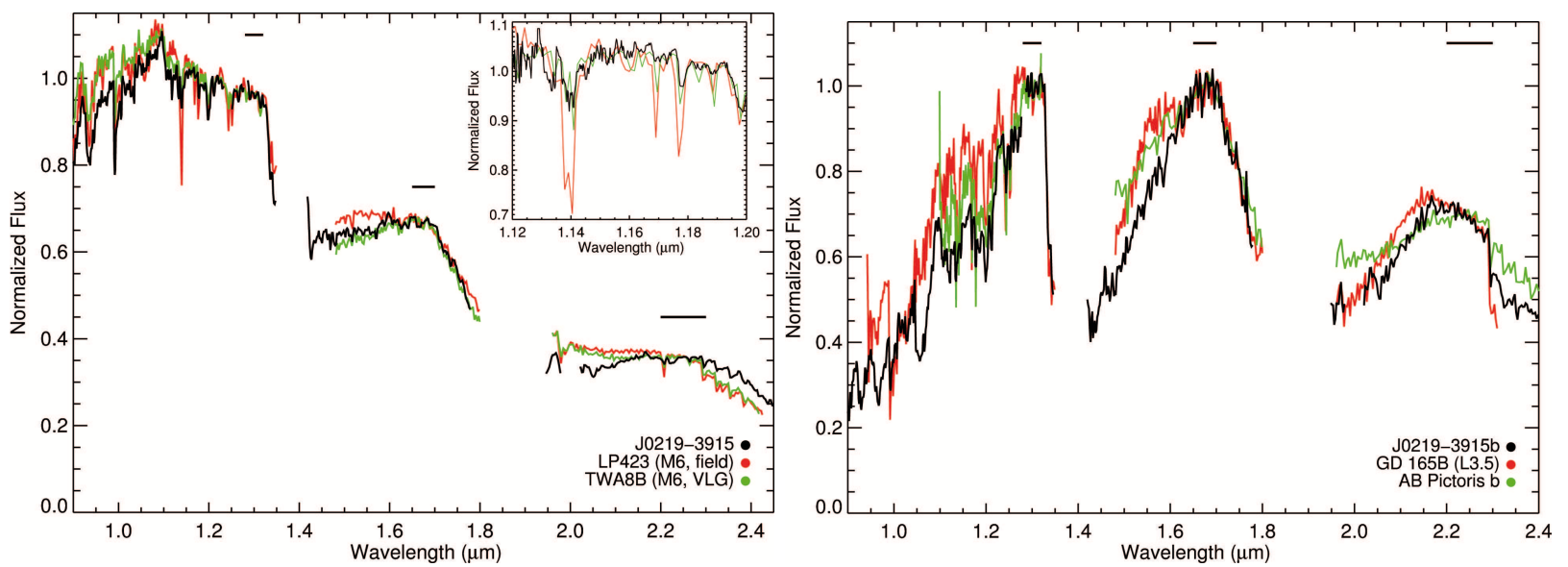

Fig. 2.- (left) Spectra of J0219-3925, compared to a field M6 dwarf (LP423) and an M6 $\gamma$ (TWA8B). Both J0219-3925 and TWA8B show an $H$-band spectrum that is more peaked that that of the higher gravity LP423. The inset shows that full resolution spectra in the $1.12-1.20 \mu \mathrm{m}$ domain; the higher gravity LP423 shows much stronger NaI absorption at $1.138 \mu \mathrm{m}$ and a stronger KI doublet at $\sim 1.17 \mu \mathrm{m}$. (right) Spectra of J0219-3925B, the young companion AB Pictoris b (data from Bonnefoy et al. 2014) and the field L3.5 dwarf GD 165 B (data from McLean et al. 2003). The $H$-band flux of J0219-3925 is noticeably more peaked than the two others, pointing toward a very low gravity (compare with middle panels in Figure 5). (both) Flux within each photometric bandpass has been normalized to that of J0219-3925 A or J0219-3925 B to highlight differences in the shape of the SED within each bandpass; normalization intervals are shown as straight black lines above spectra.

dependently as a candidate member of THA as part of the BASS survey. 2MASS and AllWISE photometry and astrometry were used to derive its membership probability to several moving groups in the Solar neighborhood. The BANYAN II tool compare the input parameters to spatial, kinematic and photometric models of young moving groups and the field population using a naive Bayesian classifier. We refer the reader to Gagné et al. (2014c) for details on this analysis. As J0219-3925 had been identified as a high probability candidate of THA, it was included in our search for distant companions before the publication of the bulk of the survey. The BANYAN II tool gives a $\mathrm{P}=99.34 \%$ probability that J0219-3925 is a member of THA. When such measurements are available, the BANYAN II tool can use the radial velocity and/or parallax to constrain membership probability further. Using the radial velocity of $10.6 \pm 0.7 \mathrm{~km} \mathrm{~s}^{-1}$ measured by Kraus et al. (2014), the THA membership probability of the host star increases to $\mathrm{P}=99.94 \%$.

We can set additional constraints on the age of this object since it displays lithium Kraus et al.
2014) and typical signs of low-gravity in its NIR spectrum (see left panel of figure 2 and Section 4.3). Within that spectral type, the presence of lithium $(\mathrm{EW}=639.9 \mathrm{~m} \AA)$ points to an age below $\sim 125$ Myr. Adding this age constraint in the BANYAN II analysis raises the THA membership probability further $(\mathrm{P}=99.93 \%)$. In addition to a membership likelihood, the BANYAN analysis provides a kinematic distance to an average precision of $\sim 10 \%$ for young moving group members (See Figure 5 in Malo et al. 2013 and Figure 8 in Gagné et al. 2014c). The kinematic distance for J0219-3925 is $39.4 \pm 2.6 \mathrm{pc}$. The $7 \%$ uncertainty includes both the contribution from the uncertainties on the proper motion of J0219 and the space velocity scatter of THA members.

As an additional confirmation, we used J02193925 apparent $I_{c}$ and $J$ magnitudes, the amplitude of the proper motion and the sky position to verify its membership probability in the BANYAN I analysis (Malo et al. 2013). It yields an a priori probability of $\mathrm{P}=96.3 \%$ of membership in THA. Similarly to the BANYAN II analysis, it is possible to include the radial velocity as an additional 
constraint in the analysis. When we do so, the probability is increased to $\mathrm{P}_{\nu}=99.9 \%$. The statistical distance inferred by this analysis is the same as predicted by BANYAN II.

\subsection{The Age of THA}

The Tucana and Horologium Associations were discovered independently by Zuckerman \& Webb 2000 and Torres et al. 2000. Further studies confirmed that what was initially considered as two associations were more likely to constitute a single young moving group to be called the TucanaHorologium association (THA; Zuckerman et al. 2001). THA is part of the so-called Great Austral Young Association (GAYA; Torres et al. 2008), including the Columba and Carina associations. Several studies (Zuckerman \& Song 2004; Torres et al. 2008; Kiss et al. 2011) proposed members of THA based on their galactic space velocity, galactic position, and signs of youth, yielding a sample of members with spectral type between A1V and early-M spawning a distance range of 36 to $71 \mathrm{pc}$. Recent studies (Rodriguez et al. 2013; Moór et al. 2013; Malo et al. 2013; Gagné et al. 2014a,b. c;; Kraus et al. 2014) proposed more than 200 strong THA candidate members, which still require more robust kinematics to confirm their membership.

The age range of THA was estimated to be $10-$ 40 Myr (Zuckerman et al. 2001; Zuckerman \& Webb 2000) based on various age indicators ( $\mathrm{H} \alpha$, lithium, HR diagram). More recently, Kraus et al. 2014 derived an average isochronal age of $30 \mathrm{Myr}$ using a new sample of 142 candidate members combined with the BCAH models Baraffe et al. 1998).

In addition to the isochronal age, the lithium depletion boundary (LDB) is a key indicator to determine the age of the low-mass star population. This method was used by Kraus et al. 2014 to determine the THA lithium depletion age of $41 \pm 2$ and $38 \pm 2$ using the BCAH and D'Antona \& Mazzitelli 1997 models, respectively.

This discrepancy between isochronal and LDB ages was already demonstrated by several studies (Song et al. 2002; Yee \& Jensen 2010; Binks \& Jeffries 2014). Recently, Malo et al. 2014a have shown that using new Dartmouth Magnetic evolutionary models (Feiden \& Chabover 2013), the isochronal age is in better agreement with the LDB age for young low-mass stars. In general, isochronal ages are revised upward with the inclusion of magnetic fields. As shown in Malo et al. (2014b), magnetic field strength measurements from highresolution spectroscopy would further constrain its age. Pending such measurement, we conservatively adopt an age range of $30-40 \mathrm{Myr}$ for the THA association, and thus, for J0219-3925 system.

\subsection{Spectral type and Gravity Indicators}

We used the method of K. Cruz et al. (in preparation; see Cruz \& Núñez 2007) to assign a spectral type to both components of J0219-3925. This method consists of a visual comparison with a grid of spectral templates while normalizing each NIR band individually. Inspecting the slope and shape of several features in each band allows to choose a template that best matches the observations. Our sequence of field, intermediate-gravity and lowgravity templates were build by median-combining various objects within each spectral types and gravity class. The spectra used to build the templates were obtained from Allers \& Liu (2013) and the SpeX Prism Spectral Libraries 1 . Both the visual comparisons of J0219-3015 and b yielded best matches to very low gravity templates; J02193015 and its companion were assigned a spectral type of M6 $\gamma$ and L4 $\gamma$ respectively. The gravity classification scheme of Allers \& Liu (2013) was subsequently used to confirm that both objects have weaker alkali lines compared to field dwarfs of the same spectral types, and that J0219-3015 B has stronger VO absorption and a triangularshaped $H$-band continuum (which was already apparent from the visual comparison). Both objects were consistently categorized as very low gravity dwarfs by this index-based classification scheme. We show that they both display typical spectroscopic signatures of a low-gravity, including lowerthan-normal alkali (K I, Na I and FeH) equivalent widths. The lower gravity causes a lower pressure in the atmosphere, which decreases both the effects of pressure broadening (responsible for the lower alkali equivalent widths) and collisioninduced absorption (CIA) of the $\mathrm{H}_{2}$ molecule. The triangular $H$-band continuum is shaped by water

${ }^{1}$ http://pono.ucsd.edu/ adam/browndwarfs/spexprism 
absorption. In the case of field brown dwarfs, the $\mathrm{H}_{2}$ CIA redirects part of the flux to the bluer side, which masks the triangular shape of the $H$-band continuum (see Rice et al. 2010 for further detail). Figure 3 illustrates the gravity-sensitive spectroscopic indices defined by Allers \& Liu (2013) for both components of the J0219-3925 system.

The intermediate gravity and very low gravity classifications generally correspond to the $\beta$ and $\gamma$ gravity classifications in the optical (Kirkpatrick 2005; Kirkpatrick et al. 2006; Cruz et al. 2009), hence we choose here to use the Greek-letter nomenclature even though our gravity classification was done in the NIR.

\subsection{Kinematics and Common Proper Mo- tion}

Astrometric measurements were performed on GMOS-S $i$-band, F2 $J$-band and SIMON imaging obtained on 2013 November 5. SIMON imaging obtained in early 2014 were not included as they were taken under poor seeing conditions $\left(\sim 2^{\prime \prime}\right)$ and do not constrain proper motion. Archival 2MASS imaging were also used; it provides only modest constraints on the PA and separation, but the long time baseline ( $\sim 15$ years) makes it a useful additions to confirm common proper motion. Astrometric errors were estimated to be at the $\sim 0$ ". 03 level for F2 and GMOS-S data and $\sim 0$ ". 1 for SIMON imaging. Astrometric measurements are shown in Figure 4. The $\chi^{2}$ for the co-moving case is 5.9 for 8 degrees of freedom. The co-moving case is equivalent to a $0.9-\sigma$ event in a Gaussian distribution while the background object model would correspond to a $6.0-\sigma$ event. While this demonstrates that J0219-3925 B is comoving to within astrometric uncertainty, any interlopers would be an L dwarf at roughly the distance of J0219-3925 with a significant proper motion that could, conceivably, match that of J0219-3925 within astrometric uncertainties. The strongest argument in favour of the two objects forming a physical pair arrises from the relative rarity of field L dwarfs per sky surface unit.

Finding a low-gravity L dwarf within $4^{\prime \prime}$ of an unrelated young $\mathrm{M}$ star is very unlikely. Considering that we started with a sample of 300 young stars, the area within a $2-6^{\prime \prime}$ annulus around these stars covers $5.6 \times 10^{-8}$ of the entire celestial sphere. With an L dwarf spatial density of $\sim 3.8 \times 10^{-3} \mathrm{pc}^{-3}$ (Cruz et al. 2007), there should be $\sim 5500 \mathrm{~L}$ dwarfs within $70 \mathrm{pc}$. The likelihood of finding a coincident $\mathrm{L}$ dwarf to a young star in our sample is about $\sim 3 \times 10^{-4}$ without considering the fact that the $\mathrm{L}$ dwarf itself also displays a low surface gravity.

Orbital motion of the pair is expected to be close to the detection threshold. With a total mass of $\sim 0.12 \mathrm{M}_{\odot}$ (see table 3 ) and a separation of $\sim 160 \mathrm{AU}$, the orbital period is on the order of $6000 \mathrm{yr}$. Assuming a face-on orbit, this leads to an orbital motion of 4 mas/yr. Orbital velocity is on the order of $800 \mathrm{~m} / \mathrm{s}$ and within reach of upcoming high-resolution infrared spectrographs such as VLT/CRIRES+ Follert et al. 2014). Both measurements are challenging but possible with existing facilities, but not with the dataset in hand. Proper constraints on the differential motion within the system will allow one to constrain whether the orbit of J0219-3925 B is consistent with a circular orbit or a highly eccentric one. Constraints on orbital eccentricity for a few systems similar to J0219-3925 could set strong limits on the plausible formation mechanisms. These measurements would provide constraints on the J0219-3925 system similar to the ones obtained for a few similar companions in the planetary-mass regime by Ginski et al. 2014 . These authors find that fitted orbits suggest that distant companions' motion is consistent with eccentric orbits; whether this holds for the J0219 3925 system remains to be determined.

\subsection{Model Fitting with Spectroscopy and Photometry}

To estimate fundamental parameters of mass, effective temperature, gravity, and radius for both J0219-3925 and J0219-3925 B we used two distinct approaches with the same set of theoretical models. First, we constrained the bulk properties of J0219-3925 A and J0219-3925 B (mass, effective temperature, surface gravity, radius and luminosity) by comparing the absolute magnitudes in each near-infrared bandpass with BT-Settl modeld2 (CIFIST 2011 opacities; Allard 2014) at 30 and 40 Myr. Absolute magnitudes were estimated from the kinematic distance; uncertainties in the kinematic distance were assumed to be $7 \%$ (see

\footnotetext{
${ }^{2}$ https://phoenix.ens-lyon.fr/Grids/BT-Settl/CIFIST2011/
} 

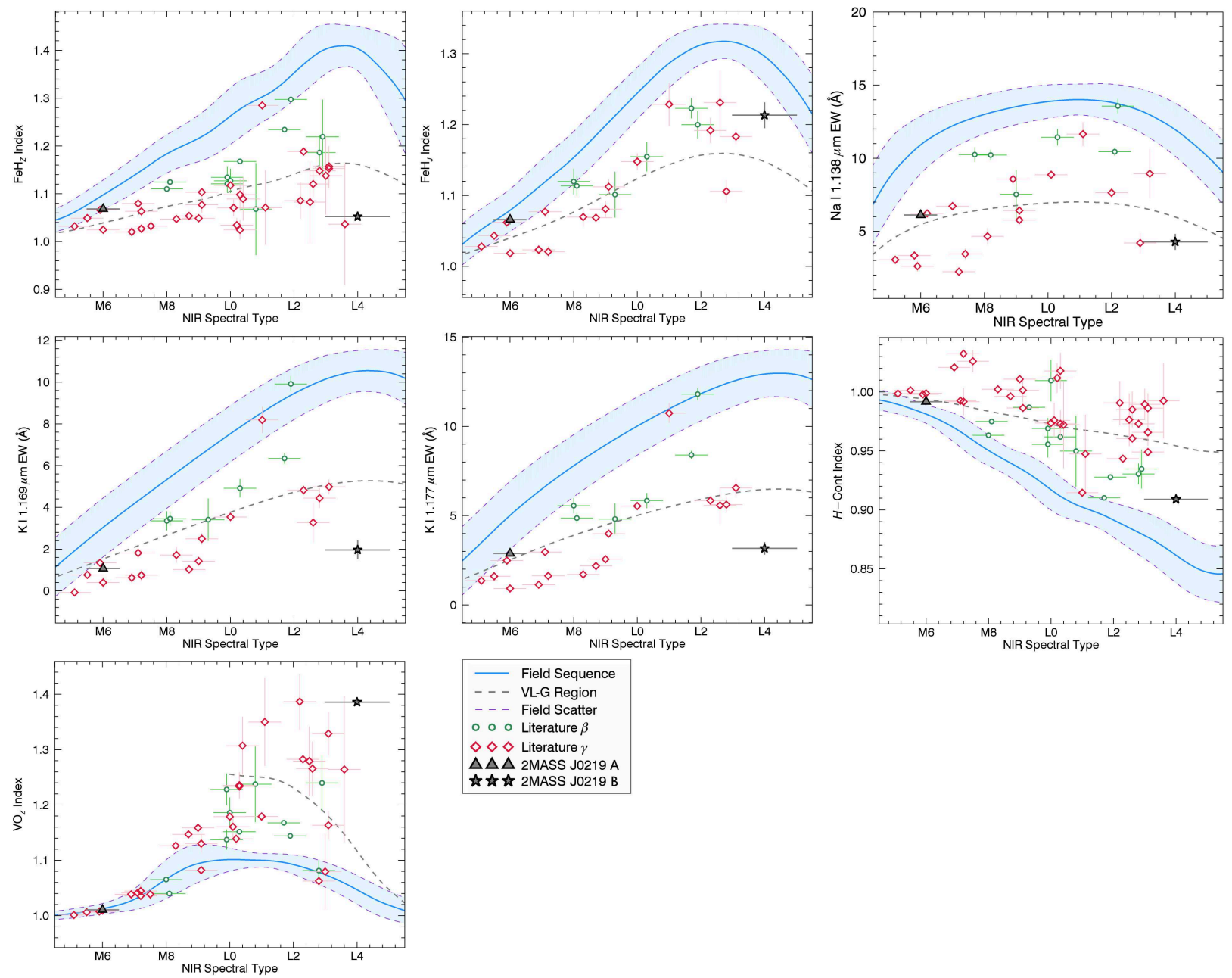

Fig. 3.- Spectral indices as defined by Allers \& Liu 2013 for J0219-3925 and J0219-3925 B, intermediate (INT-G or $\beta$ ) and very-low gravity (VL-G or $\gamma$ ) M and L dwarfs, the field sequence (thick, blue line) and its scatter (blue shaded region). The dotted line represents the delimitation between INT-G and VL-G regimes. Spectral types were offset by small $(<0.15)$ random subtypes so that vertical error bars can be distinguished. For all indices, J0219-3925 B falls outside the enveloppe of field objects, clearly highlighting its low gravity. Four indices track both metallicity and surface gravity trends $\left(\mathrm{KI}_{J}, \mathrm{FeH}_{J}, H\right.$-cont and $\left.\mathrm{FeH}_{z}\right) ; \mathrm{J} 0219-3925 \mathrm{~B}$ does not show clear signs of peculiar metallicity, following the general trends of very-low gravity members. The M6 $\gamma$ J0219-3925 falls in a part of the diagram where NIR low-gravity indices are less efficient than for its L4 $\gamma$ companion. Alkali line equivalent widths are systematically lower than field objects and consistent with very-low gravity objects. Individual objects were drawn from Allers \& Liu 2013 and Manjavacas et al. 2014. 


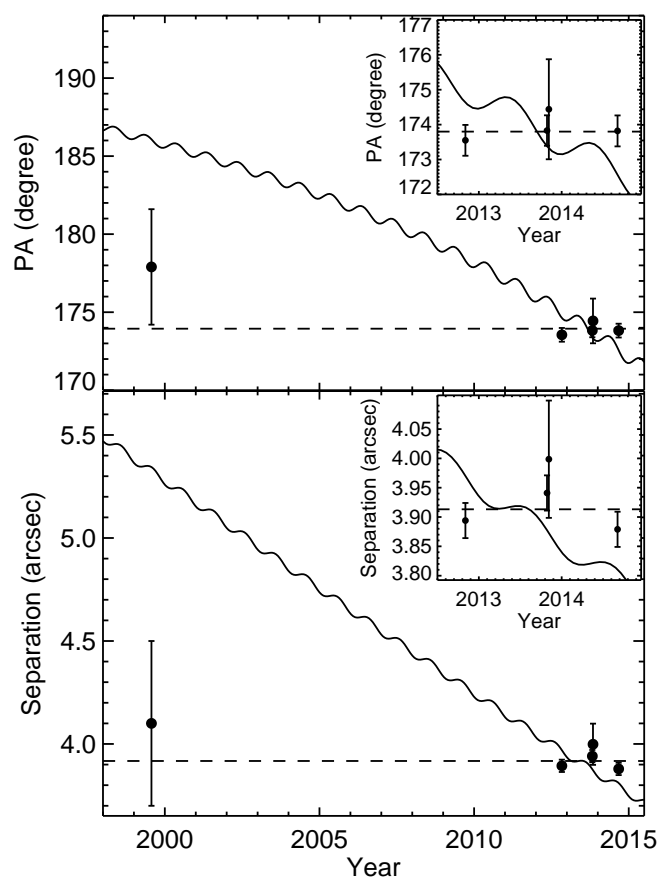

Fig. 4.- Position angle and separation for the 2MASS (1999 July 27), GMOS $i$-band (2012 Novembre 2), F2 J-band (2013 Octobre 28, 2014 Septembre 4) and SIMON J-band (2013 Novembre 5) imaging. PA and separation are constant to within astrometric uncertainties. Insets in each panel shows the astrometric measurements obtained since 2012. Dashed line shows the mean PA and separation, while the continuous line shows the expected values for a distant background object. subsection 4.1), leading to a 0.14 mag uncertainty on the absolute magnitudes. Table 3 compiles the value derived for each photometric bandpass, and the mean value for all three bandpasses is used as the best estimate. Upper and lower bounds are averaged for this best estimate, but not divided by the square root of the number of measurements as these are correlated (i.e., same age assumption, same uncertainty on distance). Overall, the mass estimate range of $12-15 \mathrm{M}_{\mathrm{Jup}}$ sets the companion at the brown dwarf/planet regime limit. Uncertainties for $\log g$ values are small (0.02 and 0.06 respectively) as the surface gravity varies very little with effective temperature for these relevant masses and the main contribution to this uncertainty arrises from the uncertainty on the age of the system. The relative uncertainty on the mass ratio (q) is smaller $(\sim 6 \%)$ than the relative uncertainty on either component's mass $(\sim 10 \%)$ as the derived masses for both components correlate through the distance and age estimates.

The second approach to constrain the properties of J0219-3925B has been to perform a $\chi^{2}$ fit between the observed and theoretical spectra in 3 individual bandpasses $\left(Y+J, H\right.$ and $K_{s}$; respectively $1.00-1.33 \mu \mathrm{m}, 1.45-1.81 \mu \mathrm{m}$ and $1.95-2.40 \mu \mathrm{m})$. For each bandpass, spectra were normalized over part of the domain (horizontal lines in Figure 5). By performing this normalization, we assume no prior knowledge of distance and absolute magnitude. The spectral fitting analysis has been performed by steps of 0.5 dex in $\log g$ between 3.5 and 5.5, and over the $1500 \mathrm{~K}$ to $1800 \mathrm{~K}$ domain. Models at intermediate gravity (4.5) better fit the SED. Within the $H$ band (middle columns), the higher-gravity (5.5) models show the distinctive flattening of the SED, which is the hallmark of old, field, L dwarfs. Within the $K$ band (right), there is a clear shift of the SED peak from $\sim 2.1 \mu \mathrm{m}$ to $\sim 2.25 \mu \mathrm{m}$ between $\log g=5.5$ and $\log g=3.5$, for all temperatures, with 2M0219-3925 B being intermediate between these scenarios. For $Y+J$ and $K$, the best-fitting model has a temperature of $1700 \mathrm{~K}$ and $\log g=$ 4.5. The value derived for $H$ is only marginally different with $T_{\text {eff }}=1600 \mathrm{~K}$ and $\log g=4.0$. These values are in very good agreement with those derived from photometry alone (see Table 3), with $T_{\text {eff }}=1683 \pm 43 \mathrm{~K}$ and $\log g=4.24 \pm 0.04$.

Overall, the mass of J0219-3925 B falls squarely 
Table 3: Constraints on properties from models.

\begin{tabular}{|c|c|c|}
\hline & J0219-3925 A & J0219-3925 B \\
\hline $\operatorname{Mass}\left(\mathrm{M}_{J}\right)$ & $114 \pm 13 \mathrm{M}_{\mathrm{Jup}}$ & $13.0 \pm 0.7 \mathrm{M}_{\mathrm{Jup}}$ \\
\hline $\operatorname{Mass}\left(\mathrm{M}_{H}\right)$ & $108 \pm 11 \mathrm{M}_{\mathrm{Jup}}$ & $13.9 \pm 1.1 \mathrm{M}_{\mathrm{Jup}}$ \\
\hline $\operatorname{Mass}\left(\mathrm{M}_{K}\right)$ & $115 \pm 13 \mathrm{M}_{\mathrm{Jup}}$ & $14.8 \pm 1.6 \mathrm{M}_{\mathrm{Jup}}$ \\
\hline Mass (mean) & $113 \pm 12 \mathrm{M}_{\mathrm{Jup}}$ & $13.9 \pm 1.1 \mathrm{M}_{\mathrm{Jup}}$ \\
\hline $\mathrm{q}\left(\mathrm{M}_{J}\right)$ & \multicolumn{2}{|c|}{$\begin{array}{c}0.113 \pm 0.007 \\
\end{array}$} \\
\hline $\mathrm{q}\left(\mathrm{M}_{H}\right)$ & \multicolumn{2}{|c|}{$0.128 \pm 0.006$} \\
\hline $\mathrm{q}\left(\mathrm{M}_{K}\right)$ & \multicolumn{2}{|c|}{$0.127 \pm 0.007$} \\
\hline q (mean) & \multicolumn{2}{|c|}{$0.123 \pm 0.006$} \\
\hline$\overline{\mathrm{T}_{\mathrm{eff}}\left(\mathrm{M}_{J}\right)}$ & $3070 \pm 73 \mathrm{~K}$ & $1615 \pm 41 \mathrm{~K}$ \\
\hline $\mathrm{T}_{\text {eff }}\left(\mathrm{M}_{H}\right)$ & $3047 \pm 84 \mathrm{~K}$ & $1686 \pm 43 \mathrm{~K}$ \\
\hline $\mathrm{T}_{\mathrm{eff}}\left(\mathrm{M}_{K}\right)$ & $3074 \pm 72 \mathrm{~K}$ & $1746 \pm 49 \mathrm{~K}$ \\
\hline $\mathrm{T}_{\text {eff }}($ mean $)$ & $3064 \pm 76 \mathrm{~K}$ & $1683 \pm 43 \mathrm{~K}$ \\
\hline log Luminosity $\left(\mathrm{M}_{J}\right)$ & $-2.22 \pm 0.06 \mathrm{~L}_{\odot}$ & $-3.92 \pm 0.03 \mathrm{~L}_{\odot}$ \\
\hline $\log$ Luminosity $\left(\mathrm{M}_{H}\right)$ & $-2.26 \pm 0.06 \mathrm{~L}_{\odot}$ & $-3.84 \pm 0.05 \mathrm{~L}_{\odot}$ \\
\hline $\log$ Luminosity $\left(\mathrm{M}_{K}\right)$ & $-2.22 \pm 0.06 \mathrm{~L}_{\odot}$ & $-3.76 \pm 0.06 \mathrm{~L}_{\odot}$ \\
\hline log Luminosity (mean) & $-2.23 \pm 0.06 \mathrm{~L}_{\odot}$ & $-3.84 \pm 0.05 \mathrm{~L}_{\odot}$ \\
\hline$\overline{l o g} g\left(\mathrm{M}_{J}\right)$ & $\overline{4} 4.59 \pm 0.06 \mathrm{~cm} \mathrm{~s}^{-2}$ & 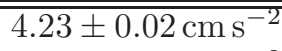 \\
\hline $\log g\left(\mathrm{M}_{H}\right)$ & $4.59 \pm 0.06 \mathrm{~cm} \mathrm{~s}^{-2}$ & $4.24 \pm 0.04 \mathrm{~cm} \mathrm{~s}^{-2}$ \\
\hline $\log g\left(\mathrm{M}_{K}\right)$ & $4.59 \pm 0.06 \mathrm{~cm} \mathrm{~s}^{-2}$ & $4.25 \pm 0.06 \mathrm{~cm} \mathrm{~s}^{-2}$ \\
\hline $\log g$ (mean) & $4.59 \pm 0.06 \mathrm{~cm} \mathrm{~s}^{-2}$ & $4.24 \pm 0.04 \mathrm{~cm} \mathrm{~s}^{-2}$ \\
\hline Deuterium $\left(\mathrm{M}_{J}\right)$ & 0 & $0.77 \pm 0.06$ \\
\hline Deuterium $\left(\mathrm{M}_{H}\right)$ & 0 & $0.68 \pm 0.07$ \\
\hline Deuterium $\left(\mathrm{M}_{K}\right)$ & 0 & $0.61 \pm 0.09$ \\
\hline Deuterium (mean) & 0 & $0.69 \pm 0.07$ \\
\hline$\overline{\text { Radius }\left(\mathrm{M}_{J}\right)}$ & $2.75 \pm 0.14 \mathrm{R}_{\text {Jup }}$ & 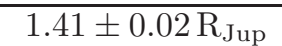 \\
\hline Radius $\left(\mathrm{M}_{H}\right)$ & $2.68 \pm 0.12 \mathrm{R}_{\mathrm{Jup}}$ & $1.44 \pm 0.04 \mathrm{R}_{\mathrm{Jup}}$ \\
\hline Radius $\left(\mathrm{M}_{K}\right)$ & $2.76 \pm 0.14 \mathrm{R}_{\mathrm{Jup}}$ & $1.46 \pm 0.04 \mathrm{R}_{\mathrm{Jup}}$ \\
\hline Radius (mean) & $2.73 \pm 0.13 \mathrm{R}_{\mathrm{Jup}}$ & $1.44 \pm 0.03 \mathrm{R}_{\mathrm{Jup}}$ \\
\hline
\end{tabular}

at the upper limit of the International Astronomical Union definition for an exoplanet - consisting of an object with a true mass below the limiting mass for thermonuclear fusion of deuterium - and is in orbit around a star, indeed J0219-3925 has a mass estimate above the hydrogen burning limit for plausible ages of THA. Evolution models predict that J0219-3925 B will have retained $\sim 70 \%$ of its initial deuterium by $40 \mathrm{Myr}$. As it is expected to have partially burned its deuterium, and considering its brightness, it would constitue a good target to spectroscopically test the brown dwarf/planet boundary, that is expected to be moderately metallicity-dependent (Spiegel et al. 2011) and its exact predicted lo- cation varies by $\sim 1 M_{\text {Jup }}$ depending on atmosphere models (Saumon \& Marley 2008). The exact nomenclature for such an object is still to be properly defined, and it joins a number of other objects such as J0103-5515(AB)b and J0122-2439 B (Delorme et al. 2013; Bowler et al. 2013) at the upper-limit of planethood. As the J0219-3925 B is expected to have burned some of its deuterium and formally lies just above the deuterium-burning limit, we use the "B" designation for stellar and brown dwarf companions rather than the "b" in usage for planetary companions even though it is predicted to be slightly less massive than objects initially described as planetary companions (e.g., AB Pictoris b). 

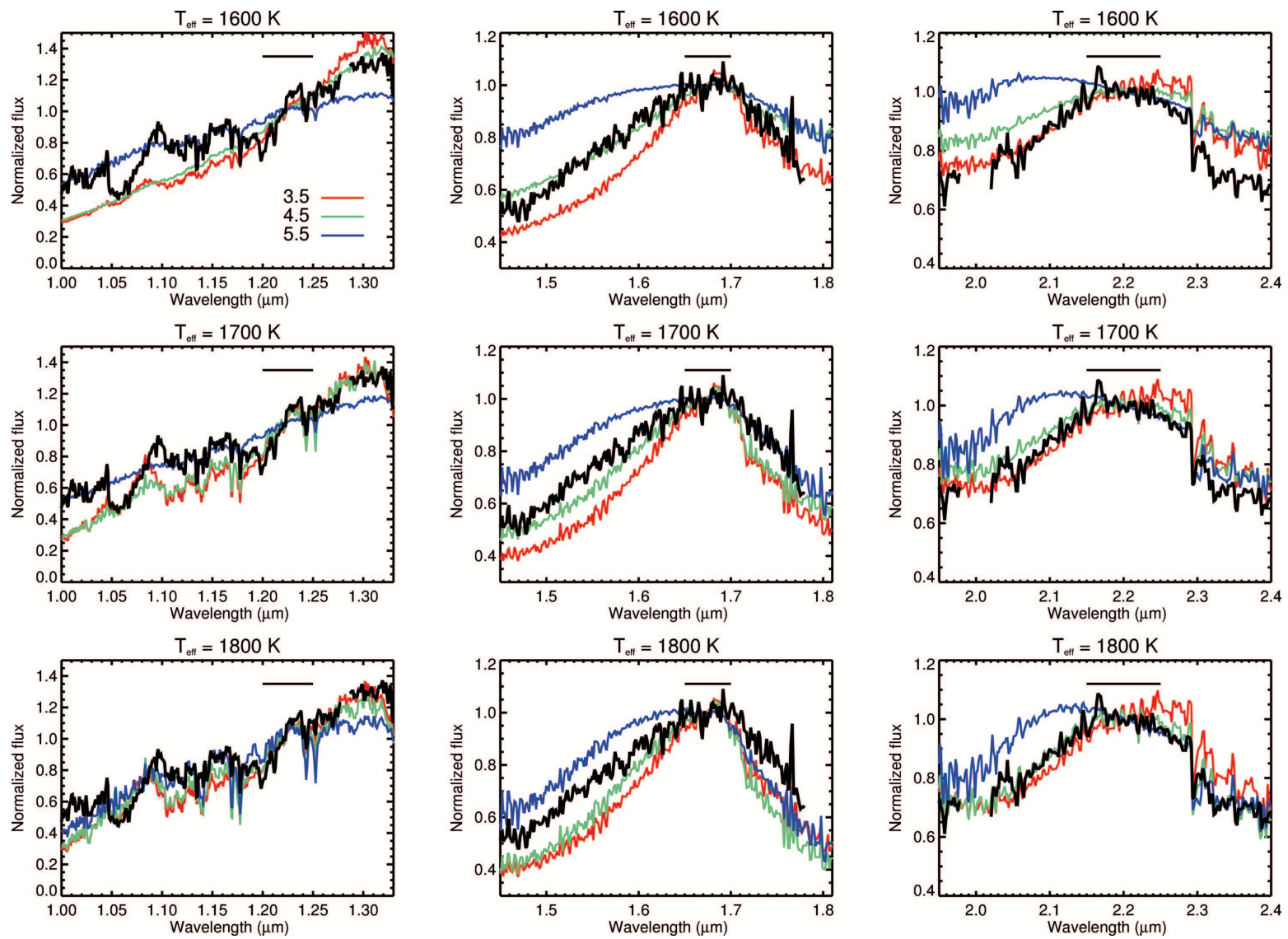

Fig. 5.- Comparison between 2M0219-3925B (black) and BT-Settl models (red, green and blue) in the $Y+J, H$ and $K$ spectral regions. To highlight shape differences in SED of individual photometric bandpasses, we show all models normalized to a common wavelength interval (shown as a thick horizontal line in each plot). For the sake of clarity, only $\log g$ values of $3.5,4.5$ and 5.5 and temperatures from $T_{\text {eff }}=1600 \mathrm{~K}$ to $1800 \mathrm{~K}$ are plotted.

\section{DISCUSSION}

Figure [6 illustrates the position of J0219$3925 \mathrm{~B}$ in the $J-K$ color-magnitude diagram compared to field ultracool dwarfs and planetary mass objects. J0219-3925 B follows the overall trend of planetary-mass companions in being redder by about $0.5 \mathrm{mag}$ than field ultracool dwarfs of similar $M_{K}$.

With a mass ratio $q=0.122 \pm 0.006$, the J0219-3925 system is similar to a heavier version of 2MASSW J1207334-393254 (Chauvin et al. 2005a), consisting of a $4-6 M_{\text {Jup }}$ planet around a $25-45 M_{\text {Jup }}$ brown dwarf (mass ratio $0.13-0.16$ ). With a separation of $>55$ AU, J1207-3932 system comes very close to J0219-3925 in terms of bind- ing energy, with a total mass $\sim 3$ times larger ( $\sim 40 \mathrm{M}_{\mathrm{Jup}}$ versus $\left.\sim 120 \mathrm{M}_{\mathrm{Jup}}\right)$, but a physical separation that is also about three times larger $(>55$ versus $>160 \mathrm{AU})$. It also comes close to the J0103-5515(AB)b system, which consists of a $12-14 \mathrm{M}_{\mathrm{Jup}}$ companion to a binary mid-M (Delorme et al. 2013). We can rule-out the possibility that J0219-3925 is a triple analogous to J0103-5515(AB)b. We only have seeing-limited observations of the system, so a hierarchical system with an inner component tighter than $\sim 0.5$ would not be resolved with the dataset at hands. The best constraints on the presence of an inner binary comes from the $M_{J}, M_{H}$ and $M_{K_{s}}$ derived from absolute magnitude versus spectral type relations for young field dwarfs. These rela- 


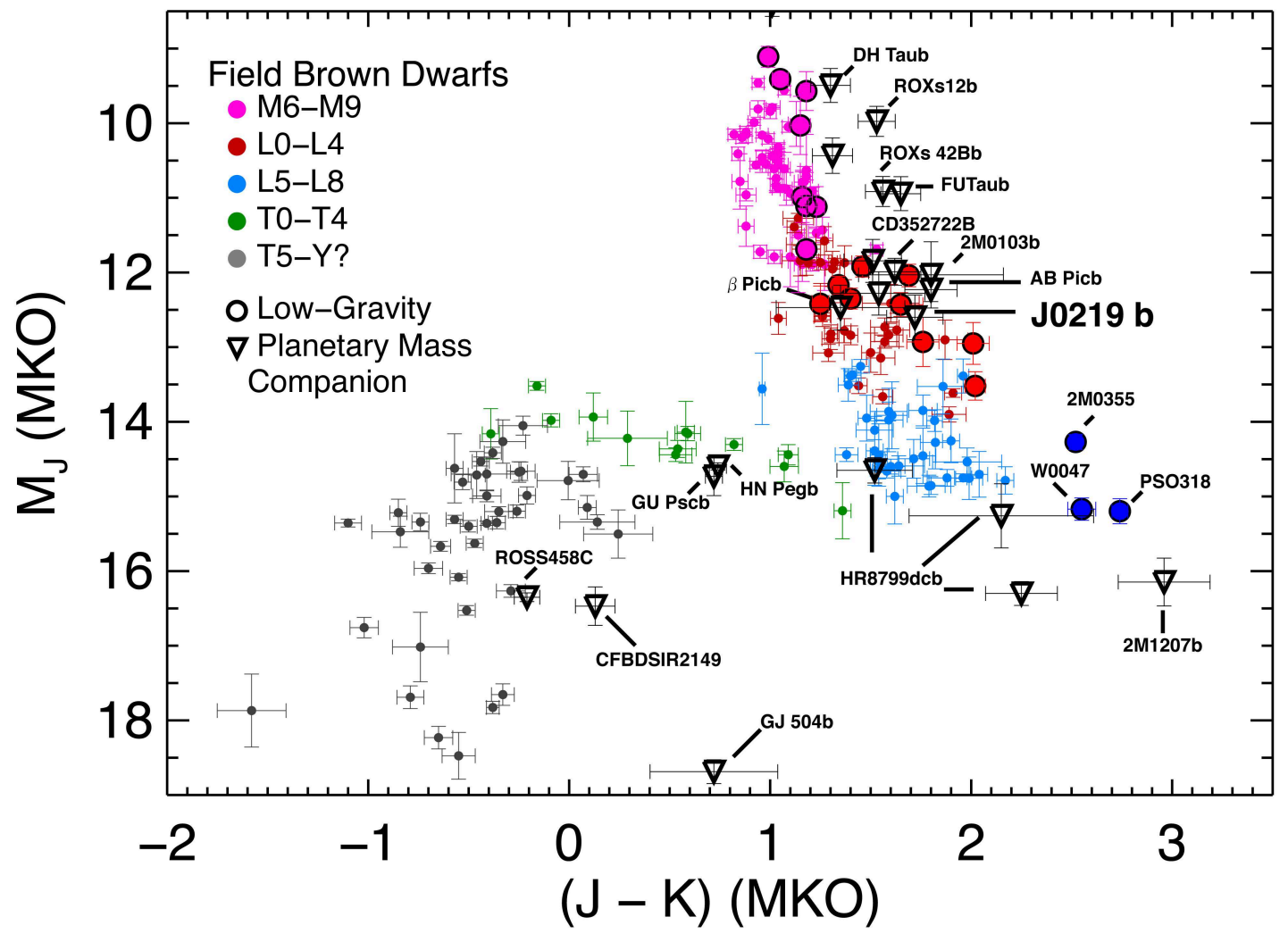

Fig. 6.- Color-magnitude plot of field dwarfs, low-gravity and directly imaged companions. J0219-3925 B is redder than typical field L dwarfs, and falls within the sequence of planetary-mass companions.

tions predict contrast ratios respectively of 4.36 , 3.78, $3.31 \mathrm{mag}$ (Gagné et al., submitted to ApJ) for single $\mathrm{M} 6 \gamma$ and $\mathrm{L} 4 \gamma$ components, and these values are in close agreement with the observed contrasts of $\Delta J=4.2 \pm 0.1, \Delta H=3.8 \pm 0.1$ and $\Delta K_{s}=3.4 \pm 0.1$. We can therefore conclude that neither J0219-3925 A nor J0219-3925 B is an equal luminosity binary. We cannot rule-out that either object is itself a high-contrast (> 1 mag) binary.

J0219-3925 B also shares various similarities with AB Pictoris b (Chauvin et al. 2005b). Both objects are companions to Tucana-Horologium members and therefore share a common age. $\mathrm{AB}$ Pictoris $\mathrm{b}$ as a slightly earlier spectral type (L0.5 \pm 0.5 ) and $\sim 0.4$ mag brighter in both $J$ and $K_{s}$ (Biller et al. 2013). The brightness difference between the two is consistent with what would be expect considering their respective spectral types (see Figure 17 in Biller et al. 2013). As shown in Figure 2, right panel, J0219-3925 B displays slightly deeper water bands, especially around $1.5 \mu \mathrm{m}$ and $1.95 \mu \mathrm{m}$ and a deeper CO bandhead longward of $2.29 \mu \mathrm{m}$, consistent with a later spectral type. This brightness difference corresponds to a mass difference of $\sim 1 \mathrm{M}_{\mathrm{Jup}}$ between the two objects and a comparative study could be used to better constrain the deuterium burning limit through high resolution spectroscopy.

Figure 7 shows the host mass versus mass ratio for imaged planetary and low-mass brown dwarf companions. Interestingly, the J0219-3925 system falls in a relative gap among known systems in that diagram. Known systems hosting a lowmass secondary $\left(<40 M_{\text {Jup }}\right)$ either have nearequal mass; many of these systems have been 
identified as near-equal luminosity field brown dwarfs or have a much smaller $q$ values (typically $q<2 \%$ ) and have been uncovered with other observing techniques. Efficient searches for nearequal luminosity sub-stellar binaries have been largely performed with HST (e.g., Burgasser et al. 2006) and laser guide star adaptive-optics imaging (e.g., Liu et al. 2012) while low-q binaries have largely been identified by other techniques such as microlensing, transit or radial velocity. Wide-field imaging surveys have also uncovered a few tens of wide companions to field stars (e.g., Baron et al. 2015, Deacon et al. 2014 and references compiled therein); many of these have mass ratios comparable to that of J0219-3925, but with significantly more massive components. The two companions uncovered that come closest to J0219-3925 are LP 261-75 and Wolf 940. LP 261$75 \mathrm{AB}$ consists of a young M4.5/L6 binary at a projected separation of $450 \mathrm{AU}$ (Burgasser et al. 2005; Reid \& Walkowicz 2006). LP 261-75 does not match the kinematic properties of young moving groups included in the BANYAN II tool, but its primary has chromospheric activity levels consistent with an age of 100-200 Myr. This youth of the system leads to a low mass estimate for the secondary of $15-30 \mathrm{M}_{\mathrm{Jup}}$. Wolf $940 \mathrm{AB}$ consists of an M4+T8.5 binary with a projected separation of 400 AU. The system has an age of 3.5-6 Gyr as derived from the chromospheric activity of the host star, leading to a mass estimate of $20-32 \mathrm{M}_{\text {jup }}$, making it a close match the LP 261-75 system albeit at an older age.

While pairs similar to J0219-3925 may be inherently rare, a relatively straightforward observation bias could explain the fact that similar systems have been overlooked. Hosts with spectral types as late as that of J0219-3925 A are in general too faint at optical wavelengths to close an adaptive-optics loop on, and are therefore under-represented in planet-search surveys. Furthermore, until very recently, very few nearby $(<50 \mathrm{pc})$ young stars that late were known, and most comparable objects had been found in significantly more distant open clusters, making the search of faint companions even more challenging. While companions similar to J0219-3925 B may exist around very nearby $\mathrm{M}$ dwarfs, the contrast ratio increases significantly with age. At an age of 1 Gyr the pair would have a contrast ratio of $\Delta J \sim 8$ and an effective temperature of $\sim 600 \mathrm{~K}$ while at $5 \mathrm{Gyr}$, it will have a contrast of $\Delta J \sim 10$ and an effective temperature of $\sim 370 \mathrm{~K}$ (Beichman et al. 2010). At these magnitudes and temperatures, J0219-3925 B will respectively have spectral types of T8.5 and Y0 (Dupuy \& Liu 2012; Marsh et al. 2013). Such companions at separations of a few arcseconds around nearby mid-M dwarfs could easily have been overlooked by current brown dwarf searches using WISE (Wright et al. 2010) or seeing-limited near-infrared observations due to the far wings of the central star's point-spread function. GAIA 3 astrometric measurements will not constrain the occurence of similar systems in the solar neighbourhood; for a face-on circular orbit and a distance of $10 \mathrm{pc}$, the host star in a system similar to J0219-3925 will display an astrometric acceleration of $\sim 1.8 \mu \mathrm{as} / \mathrm{yr}^{2}$, below the detection threshold for this mission. The GAIA mission may nevertheless uncover similar systems in young associations through their common proper motion. The best prospect to find Gyr-old siblings of the J0219-3925 system is with the use of very deep near-infrared imaging, either under good seeing and proper point-spread function subtraction, or with laser-guide star adaptive optics.

Weak constraints can be set on the occurence of companions similar to J0219-3925 B around M dwarfs for the range of separations explored here (100-5000 AU). The PALMS survey (Bowler et al. 2015) provides the largest sample to assess the abundance of analogs to J0219-3925 B, although the separation range probed with their adaptive optics observations only partially overlaps with the range of interest here. They set a $<10 \%$ upper limit on the occurence of planets between 6 and $200 \mathrm{AU}$ and $<50 \%$ limit for $1.8-570 \mathrm{AU}$ (COND atmosphere models, circular orbits and $95 \%$ confidence level). The discovery of a single object at the planetary/brown dwarf limit in our sample is therefore well within the constraints set by the PALMS survey. Our survey included stars with different levels of confirmation regarding their age drawn from different sources, so deriving clean statistical constraints on the abundance of companions similar to J0219-3925 B is non-trivial and beyond the scope of this paper. J0219-3925 was

\footnotetext{
${ }^{3}$ http://sci.esa.int/gaia/
} 
drawn from the BASS sample of late-type objects (>M4). We observed 67 high-probability BASS candidates and 13 low-probability ones (see details Gagné et al. 2015); the absence of any further candidate in the $2-6^{\prime \prime}$ separation range suggests that objects similar to J0219-3925 B are relatively rare around $\mathrm{M}$ dwarfs, with an occurence rate of $<6.8 \%$ at the $95 \%$ confidence level.

\section{Acknowledgments}

The authors thank Noel Richardson for thoughtful discussions regarding the characterization of the J0219-3925 system. This paper includes data gathered with the 6.5 meter Magellan Telescopes located at Las Campanas Observatory, Chile. Based on observations obtained at the Gemini Observatory, which is operated by the Association of Universities for Research in Astronomy, Inc., under a cooperative agreement with the NSF on behalf of the Gemini partnership: the National Science Foundation (United States), the National Research Council (Canada), CONICYT (Chile), the Australian Research Council (Australia), Ministério da Ciência, Tecnologia e Inovação (Brazil) and Ministerio de Ciencia, Tecnología e Innovación Productiva (Argentina). This publication makes use of data products from the Two Micron All Sky Survey, which is a joint project of the University of Massachusetts and the Infrared Processing and Analysis Center/California Institute of Technology, funded by the National Aeronautics and Space Administration and the National Science Foundation. The Digitized Sky Surveys were produced at the Space Telescope Science Institute under U.S. Government grant NAG W-2166. The images of these surveys are based on photographic data obtained using the Oschin Schmidt Telescope on Palomar Mountain and the UK Schmidt Telescope. The plates were processed into the present compressed digital form with the permission of these institutions.

\section{REFERENCES}

Alibert, Y., Mordasini, C., Benz, W. \& Winisdoerffer, C. 2005, A\&A, 434, 343

Allard, F. 2014, in IAU Symposium, Vol. 299, IAU Symposium, ed. M. Booth, B. C. Matthews, \& J. R. Graham, 271-272
Allers, K. N., \& Liu, M. C. 2013, ApJ, 772, 79

Baraffe, I., Chabrier, G., Allard, F., \& Hauschildt, P. H. 1998, A\&A, 337, 403

Baron, F., et al. 2015, ApJ, 802, 37

Beichman, C. A., et al. 2010, PASP, 122, 162

Biller, B. A., et al. 2013, ApJ, 777, 160

Binks, A. S., \& Jeffries, R. D. 2014, MNRAS, 438, L11

Bonnefoy, M., Chauvin, G., Lagrange, A.-M., Rojo, P., Allard, F., Pinte, C., Dumas, C., \& Homeier, D. 2014, A\&A, 562, A127

Boss, A. P. 1997, Science, 276, 1836

Bowler, B. P., Liu, M. C., Shkolnik, E. L., \& Dupuy, T. J. 2013, ApJ, 774, 55

Bowler, B. P., Liu, M. C., Shkolnik, E. L., \& Tamura, M. 2015, ApJS, 216, 7

Brandt, T. D., et al. 2014, ApJ, 794, 159

Burgasser, A. J. B., Kirkpatrick, J. D. \& Lowrance, P. J. 2005, ApJ, 129, 2849

Burgasser, A. J. B., et al. 2011, ApJS, 166, 585

Burningham, B., et al. 2011, MNRAS, 395, 1237

Burningham, B., et al. 2011, MNRAS, 414, 3590

Cameron, A. G. W. 1978, M\&P, 18, 5

Chauvin, G., Lagrange, A.-M., Dumas, C., Zuckerman, B., Mouillet, D., Song, I., Beuzit, J.-L., \& Lowrance, P. 2005a, A\&A, 438, L25

Chauvin, G., et al. 2005b, A\&A, 438, L29

Cruz, K., \& Núñez, A. 2007, in Cool Stars 17, Poster Presentation

Cruz, K. L., Kirkpatrick, J. D., \& Burgasser, A. J. 2009, AJ, 137, 3345

Cruz, K. L., et al. 2007, AJ, 133, 439

Cutri, R. M., et al. 2013, VizieR Online Data Catalog, 2328, 0

D'Antona, F., \& Mazzitelli, I. 1997, Mem. Soc. Astron. Italiana, 68, 807 


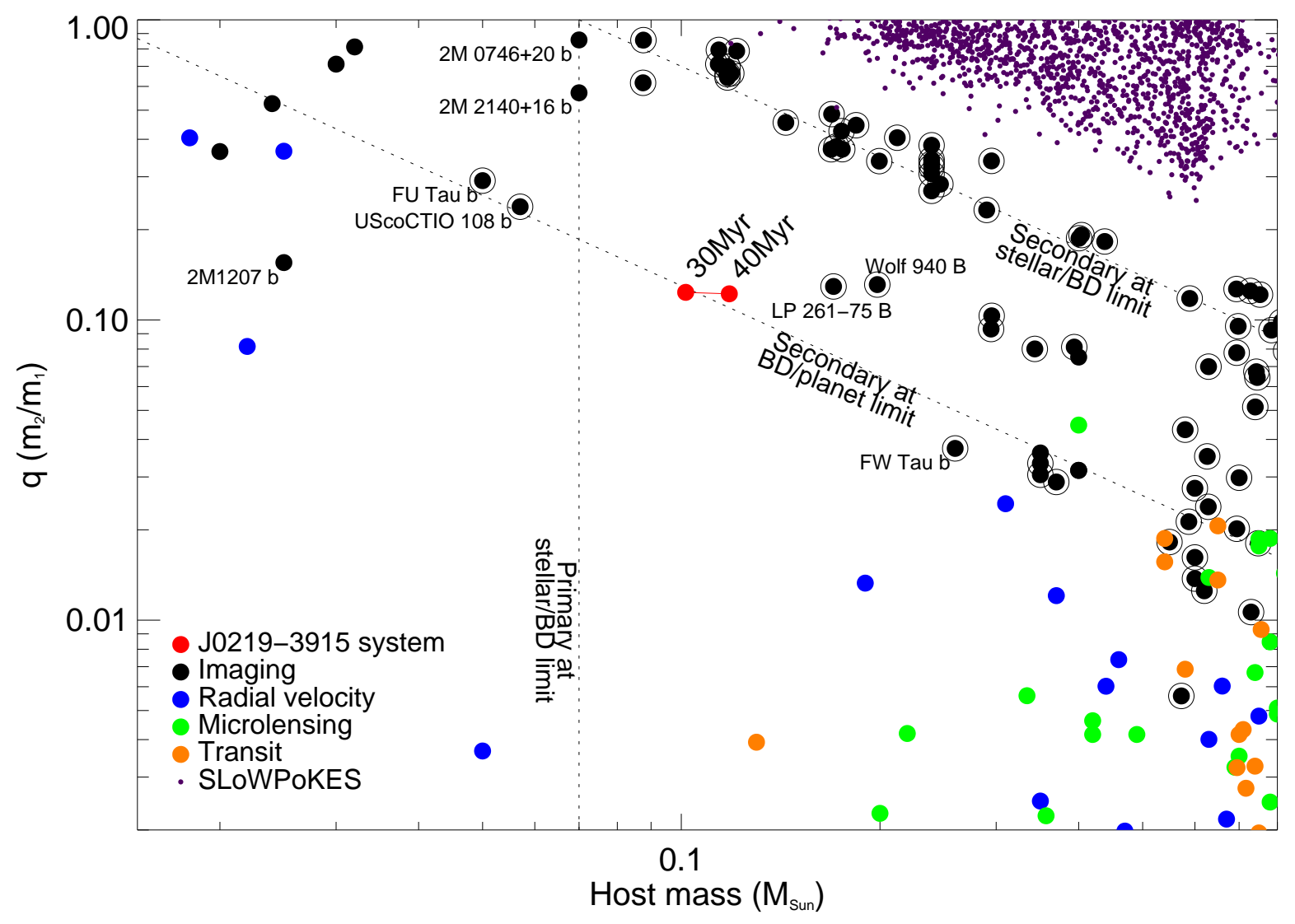

Fig. 7.- Host mass versus mass ratio for substellar companions detected through direct imaging (black), radial-velocity (blue), microlensing (green) or transit (orange). Also included is the very large sample of binaries including an cool dwarf from the SLoWPoKES survey (Dhital et al. 2010). Imaged binaries with orbital separations larger than $100 \mathrm{AU}$ are circled. Dashed lines indicate the notional $13 \mathrm{M}_{\mathrm{Jup}} \mathrm{BD} / \mathrm{planet}$ limit for companions and the brown dwarf/stellar limit for both hosts and companions and selected systems are labelled. Some hosts (e.g., 2M 0746+20; Konopacky et al. 2010) are themselves near-equal mass binaries; the total mass of the central binary is given. The position of the J0219-3925 system for ages of 30 and 40 Myr is shown. The J0219-3925 system falls in an empty part of the diagram, intermediate in properties between near-equal mass binaries such as $2 \mathrm{M} 0746+20$ and 2M 2140+16 (Konopacky et al. 2010) and planets uncovered through radial velocity measurements and imaging of young systems. Most systems close to the stellar/substellar limit are L dwarfs companions to field stars found through wide-field surveys. System parameters were drawn from the Extrasolar Planets Encyclopedia, Deacon et al. 2014, Baron et al. 2015 and Konopacky et al. 2010.

Deacon, N. R., et al. 2014, ApJ, 792, 119

Delorme, P., et al. 2013, A\&A, 553, L5

DENIS Consortium. 2005, yCat, 2263, 0

Dhital, S., West, A. A., Stassun, K. G. \& Bochanski, J. J. 2010, ApJ, 139, 2566

Doyon, R., Nadeau, D., \& Vallée, P. 2000, in As- tronomical Society of the Pacific Conference Series, Vol. 195, Imaging the Universe in Three Dimensions, ed. W. van Breugel \& J. BlandHawthorn, 548

Dupuy, T. J., \& Liu, M. C. 2012, ApJS, 201, 19

Epchtein, N., et al. 1999, VizieR Online Data Catalog, 2240, 0 
Feiden, G. A., \& Chaboyer, B. 2013, ApJ, 779, 183

Follert, R., et al. 2015, Proc. SPIE, 9147, 19

Gagné, J., Faherty, J. K., Cruz, K., Lafrenière, D., Doyon, R., Malo, L., \& Artigau, É. 2014a, ApJ, 785, L14

Gagné, J., Lafrenière, D., Doyon, R., Artigau, É., Malo, L., Robert, J., \& Nadeau, D. 2014b, ApJ, 792, L17

Gagné, J., Lafrenière, D., Doyon, R., Malo, L., \& Artigau, É. 2014c, ApJ, 783, 121

—. 2015, ApJ, 798, 73

Ginski, C., Schmidt, T. O. B., Mugrauer, M., Neuhäuser, R., Vogt, N., Errmann, R., \& Berndt, A. 2014, MNRAS, 444, 2280

Girard, T. M., et al. 2011, AJ, 142, 15

Goldman, B., Marsat, S., Henning, T., Clemens, C., \& Greiner, J. 2010, MNRAS, 405, 1140

Kalas, P., et al. 2008, Science, 322, 1345

Kirkpatrick, J. D. 2005, ARA\&A, 43, 195

Kirkpatrick, J. D., Barman, T. S., Burgasser, A. J., McGovern, M. R., McLean, I. S., Tinney, C. G., \& Lowrance, P. J. 2006, ApJ, 639, 1120

Kiss, L. L., et al. 2011, MNRAS, 411, 117

Konopacky, Q. M., Ghez, A. M., Barman, T. S., Rice, E. L., Bailey, III, J. I., White, R. J., McLean, I. S., \& Duchêne, G. 2010, ApJ, 711, 1087

Kraus, A. L., Shkolnik, E. L., Allers, K. N., \& Liu, M. C. 2014, AJ, 147, 146

Lagrange, A.-M., et al. 2010, Science, 329, 57

Liu, M.-C., et al. 2012, ApJ, 647, 1393

Manjavacas, E., et al. 2014, A\&A, 564, 55

Malo, L., Artigau, É., Doyon, R., Lafrenière, D., Albert, L., \& Gagné, J. 2014a, ApJ, 788, 81

Malo, L., Doyon, R., Feiden, G. A., Albert, L., Lafrenière, D., Artigau, É., Gagné, J., \& Riedel, A. 2014b, ApJ, 792, 37
Malo, L., Doyon, R., Lafrenière, D., Artigau, É., Gagné, J., Baron, F., \& Riedel, A. 2013, ApJ, 762,88

Marois, C., Macintosh, B., Barman, T., Zuckerman, B., Song, I., Patience, J., Lafrenière, D., \& Doyon, R. 2008, Science, 322, 1348

Marois, C., Zuckerman, B., Konopacky, Q. M., Macintosh, B., \& Barman, T. 2010, Nature, 468, 1080

Marsh, K. A., Wright, E. L., Kirkpatrick, J. D., Gelino, C. R., Cushing, M. C., Griffith, R. L., Skrutskie, M. F., \& Eisenhardt, P. R. 2013, ApJ, 762, 119

McLean, I. S., McGovern, M. R., Burgasser, A. J., Kirkpatrick, J. D., Prato, L., \& Kim, S. S. 2003, ApJ, 596, 561

Moór, A., Szabó, G. M., Kiss, L. L., Kiss, C., Ábrahám, P., Szulágyi, J., Kóspál, Á., \& Szalai, T. 2013, MNRAS, 435, 1376

Naud, M.-E., et al. 2014, ApJ, 787, 5

Padoan, P., \& Nordlund, A. 2002, ApJ, 576, 870

Pollack, J. B., Hubickyj, O., Bodenheimer, P., Lissauer, J. J., Podolak, M. \& Greenzweig, Y. 1996, Icarus, 124, 62

Reid, I. N., \& Walkowicz, L. M. 2006, PASP, 118, 671

Rice, E. L., Barman, T., Mclean, I. S., Prato, L., \& Kirkpatrick, J. D. 2010, ApJS, 186, 63

Rodriguez, D. R., Zuckerman, B., Kastner, J. H., Bessell, M. S., Faherty, J. K., \& Murphy, S. J. 2013, ApJ, 774, 101

Saumon, D., \& Marley, M. S. 2008, ApJ, 689, 1327

Simcoe, R. A., et al. 2013, PASP, 125, 270

Skrutskie, M. F., et al. 2006, AJ, 131, 1163

Song, I., Bessell, M. S., \& Zuckerman, B. 2002, ApJ, 581, L43

Spiegel, D. S., Burrows, A., \& Milsom, J. A. 2011, ApJ, 727, 57

Torres, C. A. O., da Silva, L., Quast, G. R., de la Reza, R., \& Jilinski, E. 2000, AJ, 120, 1410 
Torres, C. A. O., Quast, G. R., Melo, C. H. F., \& Sterzik, M. F. 2008, Young Nearby Loose Associations, ed. B. Reipurth, 757

Veras, D., Crepp, J. R., \& Ford, E. B. 2009, ApJ, 696,1600

Vorobyov, E. I. 2013, A\&A, 552, A129

Wright, E. L., et al. 2010, AJ, 140, 1868

Yee, J. C., \& Jensen, E. L. N. 2010, ApJ, 711, 303

Zacharias, N., Monet, D. G., Levine, S. E., Urban, S. E., Gaume, R., \& Wycoff, G. L. 2005, VizieR Online Data Catalog, 1297, 0

Zuckerman, B., \& Song, I. 2004, ARA\&A, 42, 685

Zuckerman, B., Song, I., \& Webb, R. A. 2001, ApJ, 559, 388

Zuckerman, B., \& Webb, R. A. 2000, ApJ, 535, 959

This 2-column preprint was prepared with the AAS IATEX macros v5.2. 